\title{
Contrast gain control in psychophysical contrast discrimination
}

\author{
JAMES L. DANNEMILLER \\ University of Wisconsin, Madison, Wisconsin \\ and \\ BENJAMIN R. STEPHENS \\ Clemson University, Clemson, South Carolina
}

\begin{abstract}
Contrast masking may in part reflect the operation of contrast gain control mechanisms in the visual system. Four experiments were conducted in order to examine contrast discrimination under conditions in which the base contrast was interrupted with various maskers. Interrupting the base contrast grating at $8 \mathrm{~Hz}$-with a uniform field, with a randomly phase-shifted version of the same grating, or with a higher contrast version of the same grating-interfered with contrast discrimination. These effects occurred only when the background contrast was above its own threshold. These results may be explained by assuming that the networks responsible for contrast gain control operate by temporally averaging contrast over a period greater than $62.5 \mathrm{msec}$. When this time-averaged contrast is different from the base contrast, contrast discrimination suffers.
\end{abstract}

Recent accounts of pattern masking and adaptation have proposed that their mechanisms may be related to contrast gain control (Foley, 1994; Foley \& Boynton, 1993; Greenlee \& Heitger, 1988; Ross \& Speed, 1991; Wilson, 1993). A high-contrast masking stimulus presented prior to the onset of the target is thought to force the visual system to adjust its contrast gain to accommodate a higher level of contrast. This has the effect of decreasing sensitivity for detecting contrast (see, e.g., Bowen \& Wilson, 1994), but, as Greenlee and Heitger showed, it also has the effect of increasing sensitivity for discriminating. Ross and Speed showed that a suprathreshold masker as much as $90^{\circ}$ different in orientation from a target grating shifts the contrast versus response function, as measured by the visual evoked potential (VEP), to higher levels-as if the system were adjusting its gain in all channels on the basis of the presence of high contrast at any orientation.

The suggestion that masking effects may reveal the operation of contrast gain control is based partly on neurophysiological results showing cortical contrast gain control networks. Modeling of such networks gives a prominent place to contrast gain control or contrast normalization (Albrecht and Geisler, 1991; Heeger, 1992, 1994). The response of a visual cortical neuron is determined by its sensitivity to the stimulus, normalized by the

Portions of this work were presented at the Annual Meeting of the Association for Research in Vision and Ophthalmology, Fort Lauderdale. April 1996. We thank David J. Tolhurst and two anonymous referees for very thoughtful comments and helpful suggestions for improving the manuscript. Correspondence concerning this article should be addressed to J. L. Dannemiller, Department of Psychology, University of Wisconsin. 1202 W. Johnson St. Madison, WI 53706 (e-mail: jldannem@facstaff.wisc.edu). pooled responses of many other neurons (Heeger, 1992; Tolhurst \& Heeger, 1997). In particular, activity in a large pool of neurons can suppress the response of an individual neuron. An example of such an effect is cross-orientation masking (Bonds, 1989), in which the response of an orientation-selective visual cortical neuron is suppressed by other contrast stimuli that are as much as $90^{\circ}$ different in orientation from the neuron's preferred orientationstimuli that normally evoke little response from the neuron because of its orientation selectivity.

Most of the physiological examples used to support contrast normalization show the nonspecific suppressive effects of stimuli presented simultaneously with a target stimulus (see, e.g., Geisler \& Albrecht, 1992). Bonds (1993) showed, however, that contrast gain control has a temporal component as well; the responses of visual cortical neurons were shown to be heavily influenced by the recent $(<3 \mathrm{sec})$ temporal history of contrast within the neuron's receptive field. When a given contrast had been preceded by a series of lower contrasts, the response of the neuron was larger than when that same contrast had been preceded by a series of higher contrasts. Bonds interpreted this result as showing that these cortical neurons exhibited a form of contrast gain control; the contrast gain of a target neuron was adjusted by the recent history of contrast across an inhibitory network of neurons. This is very similar to the notion of a pool of neurons contributing a divisive signal to adjust the contrast response of an individual neuron (Foley, 1994; Heeger, 1992).

How long does it take contrast gain control to exert its effects? In Heeger's model of contrast normalization, the divisive operation that provides the normalization signal contains a temporally low-pass filter to avoid instability in the neuron's output. Such a low-pass filter provides the 
equivalent of a time-averaged contrast gain control signal. Although Heeger left the time constant of such a filter unspecified, we can look to other work to determine its likely values. Geisler and Albrecht (1992) showed that contrast gain control acts very quickly in cat cortical neurons, probably reaching full strength within several hundred milliseconds. Bonds (1993) showed that cortical gain control is evident with even 50 -msec pulses of contrast but that higher contrast pulses as long as $750 \mathrm{msec}$ may be necessary to reduce the response of a neuron to $50 \%$ of its baseline level. Wilson and Humanski (1993) include contrast gain control in their model of psychophysical contrast adaptation. The gain control process requires approximately $200 \mathrm{msec}$ to approach completion in their model. The gain control in Wilson's (1993) masking study required approximately $150 \mathrm{msec}$ to become fully effective. Foley and Boynton (1993) showed that the desensitizing effects of a forward masker disappeared when approximately $200 \mathrm{msec}$ intervened between the offset of the masker and the onset of the target. All of these studies agree that there are rapid gain control processes (different from longer term contrast adaptation) that adjust the responses of single neurons or the visual system as a whole to the recent temporal history of contrast.

How might contrast gain control be implemented in the visual system? Most models suggest that gain may be changed dynamically by altering the half-saturation contrast--the contrast at which the neuron's response reaches half its maximum, saturated response (see, e.g., Greenlee \& Heitger, 1988; Ross \& Speed, 1991; Wilson, 1993; Wilson \& Humanski, 1993). Such a suggestion is supported by neurophysiological data such as those presented by Geisler and Albrecht (1992). A null adapting grating that by itself evokes little response from a neuron nonetheless shifts the contrast versus response function of the neuron to higher contrast levels (rightward on a contrast versus response plot) as the null adaptor's contrast is increased. Such a shift is exactly what would be predicted from a gain control mechanism that attempted to maintain differential sensitivity to contrast despite a limited dynamic range. As Heeger (1992) noted, shifting a neuron's half-saturation contrast is equivalent to rescaling the input contrast. Notice that this type of contrast-set gain control differs from response-set gain control, in which gain is changed inversely in proportion to the system's response to the masking or adapting grating (Foley \& Boynton, 1993).

The experiments to date that have been used to support the hypothesis that contrast gain control plays a role in masking or adaptation have used high-contrast masks, presented simultaneously with, or briefly before, the target stimulus. The general effect is that these high-contrast masks raise the contrast threshold, presumably by forcing the half-saturation contrasts of the neurons responsible for detecting the target to higher contrast levels. As noted above, this also has the adaptive effect of improving incremental contrast sensitivity at higher base con- trasts. Consider the predictions of this contrast gain control model for an observer who is asked to detect an increment over a base contrast when the system is forced to adjust to contrasts lower than the base contrast. The base contrast will now fall on a more saturated portion of the contrast-response function (CRF) producing poorer incremental contrast sensitivity relative to conditions in which the system has adjusted its gain to match the base contrast level. If this model is correct, incremental contrast sensitivity should deteriorate when lower contrast masks force the system to adjust to contrast levels below the base contrast.

\section{EXPERIMENT 1}

The purpose of the first experiment was to determine the effects on contrast discrimination of forcing the system to adjust its contrast gain to match a level below the base contrast. We did this by periodically interrupting the base contrast grating with a uniform field. Given some temporal averaging of contrast to set the contrast gain, one would predict from the models discussed above that the visual system would adjust contrast gain to a value lower than the base contrast. This, in turn, should cause contrast discrimination to deteriorate. The zero-contrast masker in this case appeared both before and after the signal contrasts.

\section{Method}

Subjects. Fourteen college-age undergraduate students served as observers. All were naive as to the experimental purpose. Each observer furnished six thresholds-one in each of the six stimulus conditions described below. All the observers had normal or corrected-to-normal visual acuity and wore their corrections during testing. The observers were given practice trials to acquaint them sufficiently with the procedure.

Apparatus and Stimuli. Vertical sine-wave gratings were computer generated using a Vision Metrics PC Pattern Generator, resident in an IBM PC, and displayed on an INFAX (DM/9B) composite monitor. The PC Pattern Generator controls signal contrast through a linear attenuator ( 128 levels) and an independent logarithmic attenuator (256 levels) that are cascaded to allow precise contrast control. Cascading these attenuators provided 32,768 possible luminance levels. The monitor was calibrated using a UDT (151) photometer. The maximum calibrated contrast on the monitor was 0.72 . The base contrast for all of the gratings in the first experiment was 0.10 , and the mean luminance was $68 \mathrm{~cd} / \mathrm{m}^{2}$. Observers viewed the display from a distance of $167 \mathrm{~cm}$. The gratings were presented through a $5.0^{\circ} \times 3.9^{\circ}$ aperture, and the surround was dark.

Spatial frequencies of $0.8,1.6$, and $3.2 \mathrm{cpd}$ were used, corresponding to 4,8 , and 16 cycles filling the $5.0^{\circ}$ width of the display. Displays were presented at central fixation under two temporal conditions: (1) interrupted--the test grating $(C$ or $C+\Delta C)$ alternated at a temporal rate of $8 \mathrm{~Hz}$ (square-wave) with a uniform field at mean luminance--and ( 2 ) continuous - the test grating ( $C$ or $C+$ $\Delta C$ ) alternated at $8 \mathrm{~Hz}$ with a grating whose contrast was $C$. Thus, in the continuous condition, the base contrast grating was presented continuously for $1 \mathrm{sec}$, and the grating with the incremented contrast (target) was presented as eight periodic contrast increment pulses $(\Delta C$ ) over the base contrast $(C)$. See Figure 1 for the temporal conditions of stimulus presentation. We note here that the reti- 
C

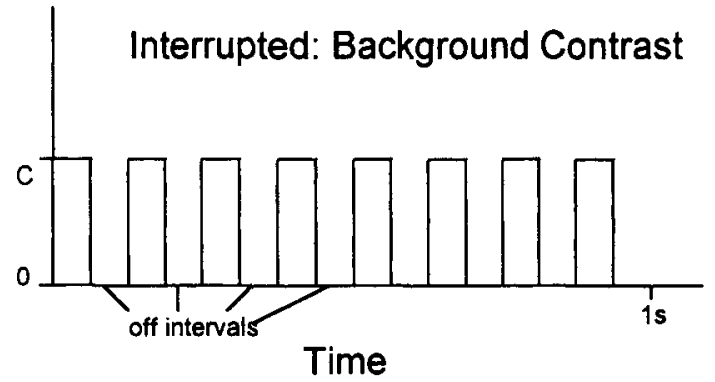

C

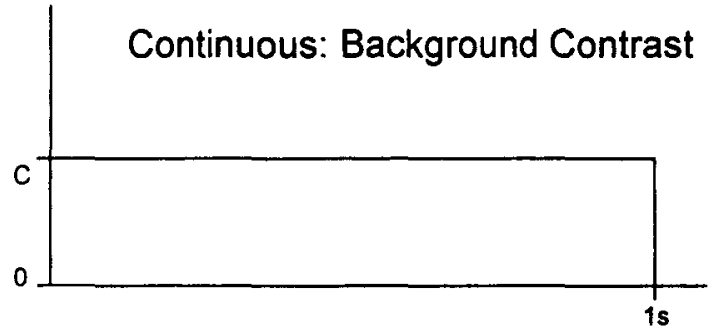

Time
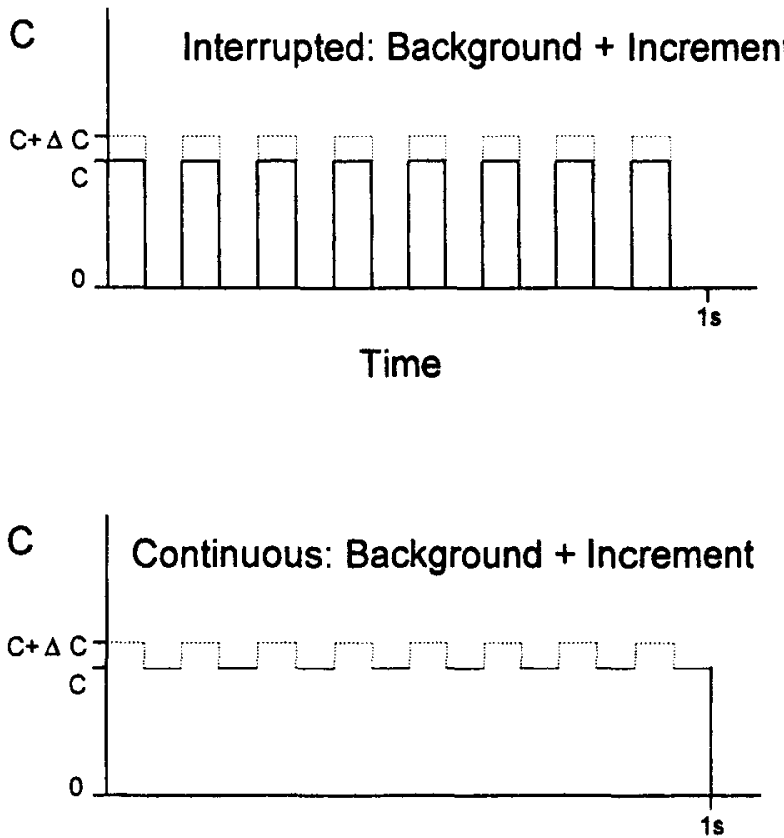

Time

Figure 1. Stimulus conditions. Left panels: Base contrast intervals $(C)$. Right panels: Target intervals $(C+\Delta C)$. Top panels: $I n$ terrupted - The base contrast grating was interrupted at $8 \mathrm{~Hz}$ by a uniform field at mean luminance. The target interval contained the higher contrast grating also interrupted at $8 \mathrm{~Hz}$ by the uniform field. Bottom panels: Continuous-The base contrast grating was continuously exposed throughout the 1-sec display interval. The target interval contained contrast increments of $\Delta C$ pulsed at $8 \mathrm{~Hz}$. The $y$-axis in each panel shows the contrast over time; the mean luminance remained constant across all intervals.

nal information for the contrast discrimination is identical in the interrupted and continuous conditions; it is only the half cycles in which the base or target gratings are presented that contain the information for the discrimination, and these periods are strictly equal in the two conditions. The other half cycles were identical across the two test intervals in each trial; they contributed no information relevant to the discrimination.

Design and Procedure. The observer's task in all conditions was to choose the grating that had the greater contrast. A twotemporal-interval forced-choice procedure was used to display the gratings. One interval contained the grating at contrast $C$, and the other interval contained the grating at contrast $C+\Delta C$. Each interval was $1 \mathrm{sec}$ long, and the intervals were separated by $1 \mathrm{sec}$. The spatial phase of the grating with respect to the center of the display was randomized before each trial, to reduce potential effects associated with long-term, local luminance adaptation. The contrast difference between the gratings was controlled by a three-down, oneup staircase, with a step size of $2.0 \mathrm{~dB}$. The initial value of $\Delta C$ was set by the observer to a contrast level noticeably above threshold. Six reversals were used to estimate increment threshold by averaging the logs of the reversal contrasts. The order of spatial frequency and the two temporal conditions (interrupted vs. continuous) were counterbalanced within and across subjects.

\section{Results and Discussion}

Figure 2 shows the average contrast increment thresholds and their standard errors at all three spatial frequencies in the continuous and interrupted conditions. Interrupting the background contrast at $8 \mathrm{~Hz}$ with a uniform field increased the contrast increment thresholds significantly at the two lower spatial frequencies, but it produced no significant effect at the highest spatial frequency.

The results of Experiment 1 at the two lower spatial frequencies would be predicted by models of contrast gain control that assume that (1) contrast gain control maximizes sensitivity to incremental contrast around the base contrast level and (2) the signals that adjust contrast gain involve a low-pass temporal filter that effectively averages contrast over a period greater than $62.5 \mathrm{msec}$. These results provide support for the contrast gain control model of masking, if we assume that interrupting the base contrast grating at $8 \mathrm{~Hz}$ with a uniform field forces the system to set its contrast gain to match a level below the base contrast (shifting the CRF leftward on the contrast axis). The base and target contrasts then fall at a more saturated portion of the CRF, requiring a larger increment in contrast to produce a criterion response change (see Greenlee \& Heitger, 1988, and Wilson, 1993, for similar arguments).

\section{EXPERIMENT 2}

We examined the differences that we observed in Experiment 1 between the continuous and the interrupted conditions more closely in Experiment 2 by collecting data from 2 observers over a more extensive range of back- 


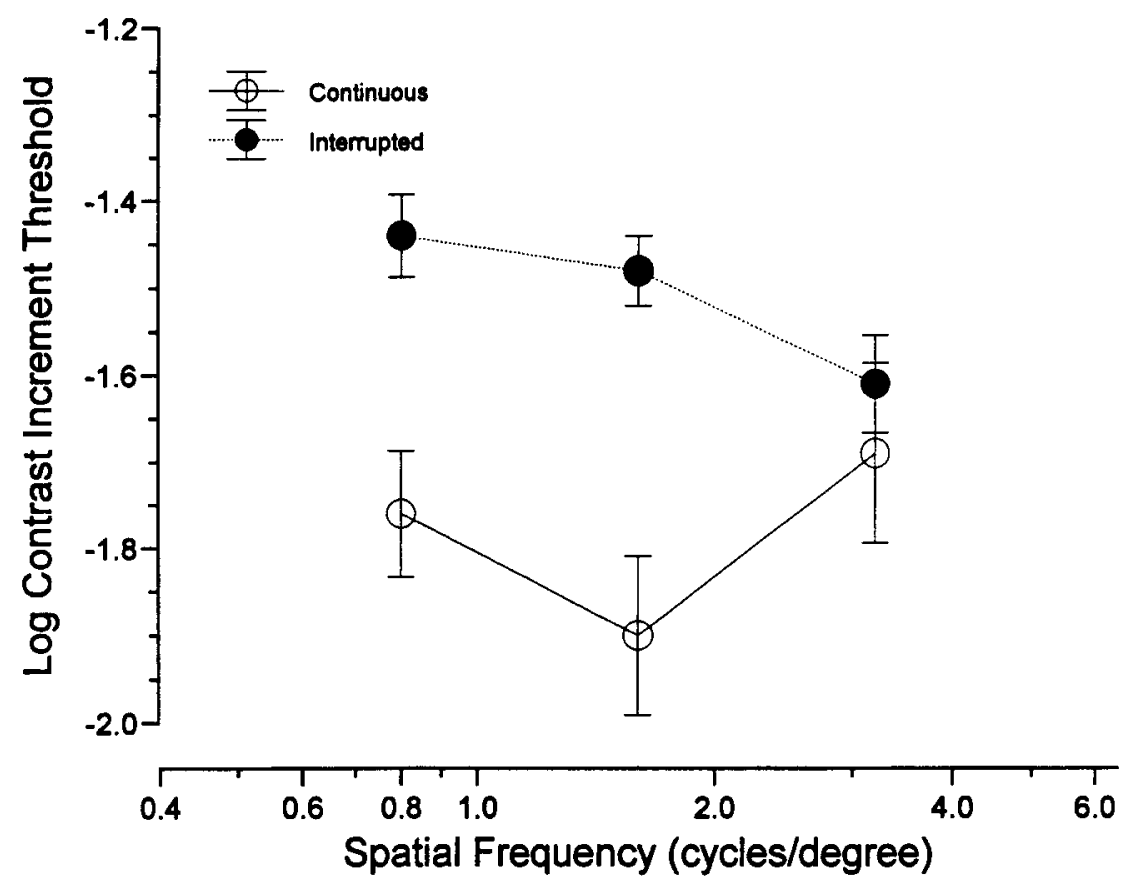

Figure 2. Results from Experiment 1. Mean contrast increment thresholds ( $y$-axis) plotted versus spatial frequency for continuous (open symbols) and interrupted (closed symbols) conditions. The background contrast was $10 \%$. Error bars are $\pm 1 S E M$.

ground contrasts. This allowed us to determine the effects of interrupting the base contrast on the slopes of threshold versus contrast (tvc) curves.

\section{Method}

Subjects. Two practiced observers furnished four to six increment threshold estimates in each of the 12 conditions described below.

Apparatus and Stimuli. Vertical gratings were generated as described above. The two observers viewed the 0.8 -cpd gratings from $84 \mathrm{~cm}$.

Design and Procedure. Thresholds were obtained in 12 conditions: 2 temporal display conditions (interrupted and continuous; see Experiment 1 , above) $\times 6$ base contrasts (ranging from 0 to 0.60 ). Each observer provided $4-6$ threshold estimates per condition, and these estimates were averaged to obtain the final thresholds shown below. All other testing procedures were identical to those described in Experiment 1.

\section{Results and Discussion}

The results are shown in Figure 3. The thresholds in the continuous and interrupted conditions were similar at low-background contrasts. At higher background contrasts, thresholds rose more quickly in the interrupted condition than in the continuous condition. The slopes of the rising portions of the contrast discrimination functions over the three highest contrasts ${ }^{\prime}$ for the two observers were .80 and .81 in the interrupted condition and .44 and .35 in the continuous condition for observers E.S. and M.N., respectively. These slopes are very similar to those in Wilson (1993) for two conditions in which gain control was ( 1 ) given enough time to exert its effects (slope =
$0.4)$ and (2) not given enough time to exert its effects (slope $=0.7)$. At the highest contrast $(60 \%)$, the contrast increment threshold was lower by a factor of approximately three in the continuous condition, relative to the interrupted condition. The lower slopes in the continuous conditions are consistent with Greenlee and Heitger's (1988) suggestion that adaptation works to linearize the system's response to contrast in the region near the prevailing contrast. A completely linear system would require a constant $\Delta C$ at all base contrasts.

There is some indication in the data from these $2 \mathrm{ob}-$ servers that the location of the dipper may also be changing between the interrupted and continuous conditionsas if the two curves were shifting relative to each other on the contrast axis. We cannot be sure that this is the case from these data, so we will leave its interpretation until we have additional data.

\section{EXPERIMENT 3}

The interrupted conditions in the experiments above contained half cycles in which the contrast in the pattern was set to zero. Consider these half cycles to have been "filled in" with the base contrast grating to create the continuous conditions. The gain control model predicts that this allows the system ample time to adjust its contrast gain to the base contrast level. Models of contrast normalization (Heeger, 1992) and masking-as-gain-control (Foley, 1994; Ross \& Speed, 1991) assume that this gain control is fed by a network of broadband (in spatial fre- 

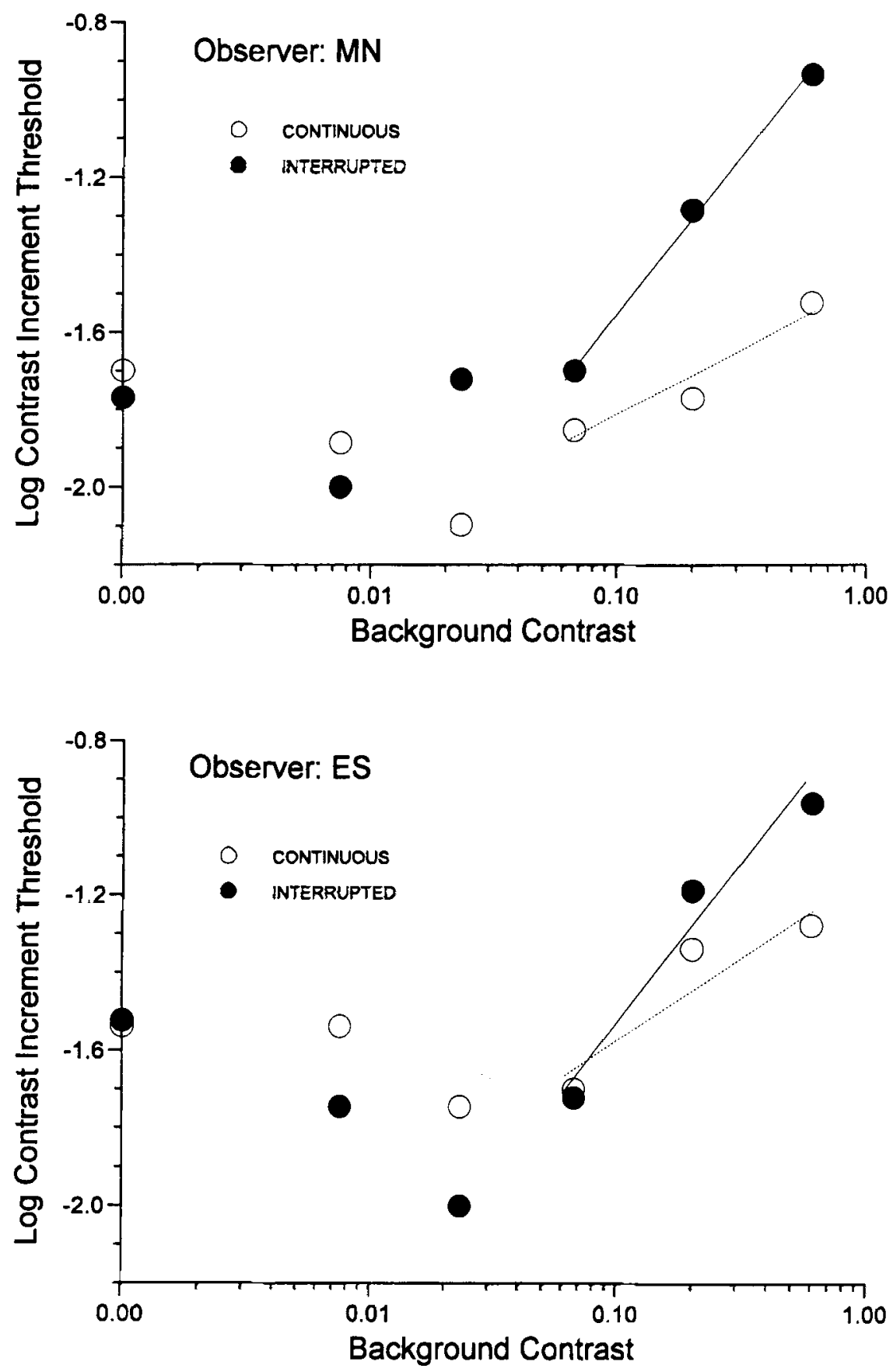

Figure 3. Mean contrast increment thresholds as a function of background contrast for two observers (M.N., top panel, and E.S., bottom panel). Open symbols - continuous background contrast; closed symbols - interrupted background contrast. The spatial frequency was $0.8 \mathrm{cpd}$. Best-fitting straight lines over the three highest contrasts are also shown. Each point is based on an average of four to six different threshold estimates. Error bars are approximately the sizes of the symbols.

quency and orientation) units. A prediction from these models is that the phase of the masker relative to the target should be unimportant in determining its masking effects if the masker has the same spatial frequency as the target; only the absolute contrast should matter. This is especially true in quadrature models such as Heeger's.
Some support for this comes from a study by Foley and Boynton (1993). They showed equal masking effects on contrast threshold for maskers that had no temporal variation and maskers that phase alternated (square wave) at $15 \mathrm{~Hz}$. Both of these maskers have the same absolute contrast over a cycle, so both should have been equally ef- 


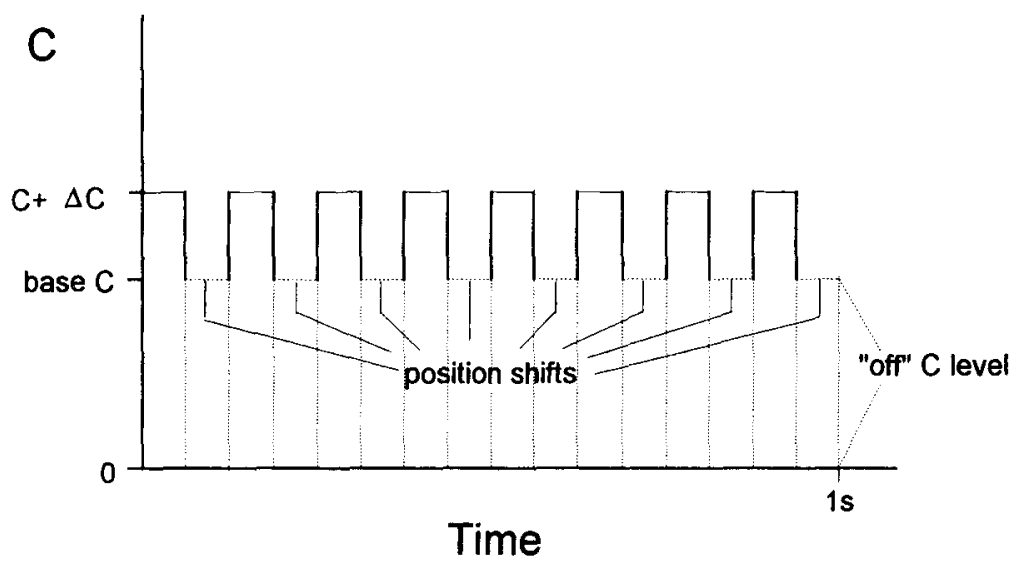

\begin{tabular}{|c|c|c|}
\hline \multicolumn{2}{|c|}{ Background Contrast } & $C=0.2$ \\
\hline \multicolumn{2}{|c|}{$\begin{array}{l}\text { CONTINUOUS: } \\
\text { INTERRUPTED }\end{array}$} & $\begin{array}{l}C \text { level }=0.2 \\
C \text { level }=0.0\end{array}$ \\
\hline $\begin{array}{l}\text { FIXED: } \\
\text { RANDOM: }\end{array}$ & $\begin{array}{l}\text { Phase (p } \\
\text { Phase ( } p\end{array}$ & $\begin{array}{l}\text { tion) shift }=0 \text { deg } \\
\text { tion) shift }=\text { Random (range } \pm 180 \text { deg })\end{array}$ \\
\hline Target: & $\Delta C>0.0$ & NonTarget: $\Delta C=0.0$ \\
\hline
\end{tabular}

Figure 4. Stimulus conditions for Experiment 3. The continuous and interrupted conditions described for Experiment 1 were replicated here in the fixed condition. In the random condition, the position of the grating within the viewing window was randomly perturbed (range $\pm 180^{\circ}$ phase) at the midpoint of each off cycle. During the off cycle in the interrupted condition, the screen was a uniform field, so these jumps were not evident until the next on cycle. During the off cycle of the continuous condition, the base contrast grating was present, so these jumps were evident to the observer. The contrast pulses in the continuous condition lagged the random phase changes by $90^{\circ}$ phase ( $\left.31.25 \mathrm{msec}\right)$.

fective in forcing a contrast-set gain control mechanism to adjust to that contrast. The next experiment tested this prediction further by randomizing the phase of the grating on every cycle. Whether the grating remains fixed in phase throughout these cycles (continuous conditions above) or appears in random phases, the absolute contrast is the same. A contrast-set gain control system should adjust equivalently to both of these stimuli.

\section{Method}

Subjects. Twenty-six college-age subjects provided one threshold estimate in each of the four conditions described below. All were naive as to experimental purpose. All the observers had normal or corrected-to-normal visual acuity and wore their corrections during testing.

Apparatus and Stimuli. Vertical gratings were displayed as described above. The base contrast of the 0.8 -cpd gratings was fixed at 0.20 .

Design and Procedure. Threshold estimates were obtained from each observer in four conditions: two temporal display conditions (interrupted and continuous; see Experiments 1 and 2, above) $\times$ two phase conditions (fixed and random). In the fixed phase condition, the phase of the grating remained fixed during each of the two display intervals. This condition was identical to those described in Experiment 1, above. In the random condition, the spa- tial phase of the grating with respect to the center of the display was shifted randomly over a range of $\pm 180^{\circ}$ during each cycle of the 8 $\mathrm{Hz}$ temporal modulation. In other words, the grating shifted phase randomly and nearly instantaneously eight times during each of the I-sec display intervals on a given trial. The phase shifts occurred during the off half of each cycle. In the interrupted condition, this meant that the position of the grating was shifted during the half of each cycle in which the uniform field was displayed. Thus, the observers did not actually see the shift when it occurred but only after the grating reappeared at the beginning of the next on period. In contrast, in the continuous condition, this phase shift occurred eight times during each $1-\mathrm{sec}$ display interval when the grating was at its base contrast. Thus, these phase shifts were visible in the continuous conditions of this experiment. See Figure 4 for a schematic of the temporal relationships between the contrast increment pulses and the phase shifts.

\section{Results and Discussion}

The results of Experiment 3 are shown in Figure 5. The fixed/random manipulation produced no effect in the interrupted condition (solid squares). Randomly shifting the phase eight times during the off period, when the grating was not visible, did not cause an increase in threshold. Forcing the observer to monitor the contrast across many different phases (jittered-phase condition) rather 


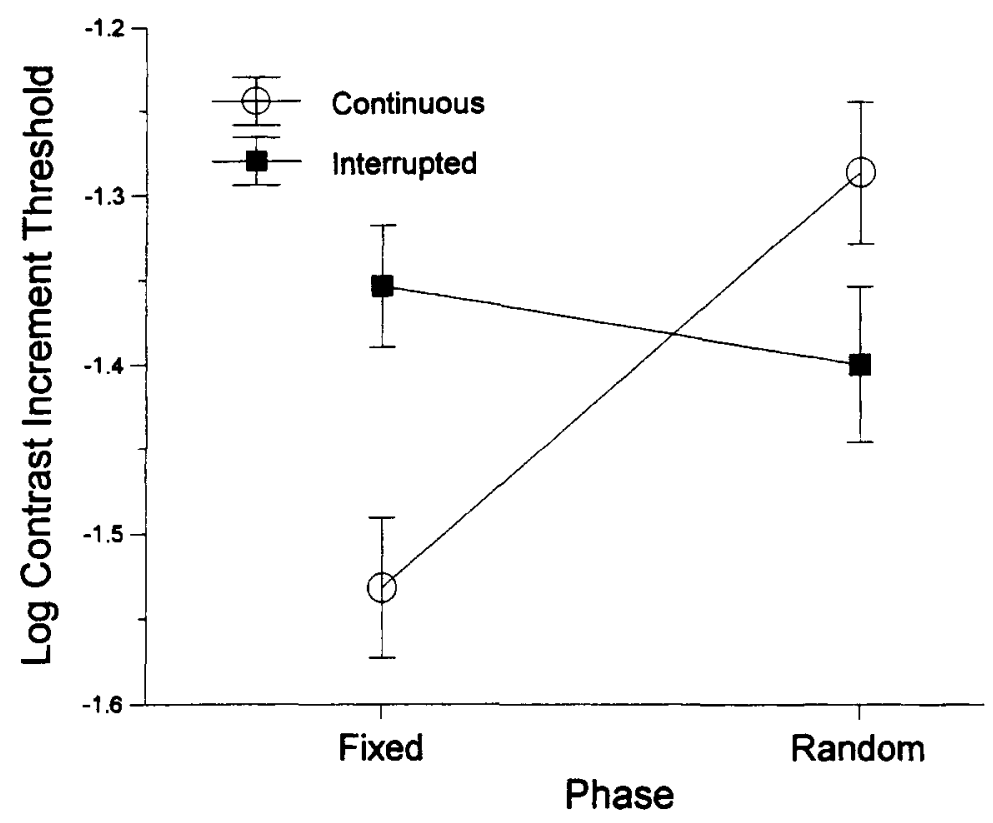

Figure 5. Mean contrast increment thresholds ( $\pm 1 S E M$ ) from Experiment 3 . The spatial frequency of the grating was $0.8 \mathrm{cpd}$ and its contrast was $20 \%$. Solid squares: interrupted background contrast; open circles: continuous background contrast (see Figure 4 for stimulus details). Fixed: The phase of the grating remained fixed throughout each display interval. Random: The phase of the grating was randomly shifted within a $\pm 180^{\circ}$ range at $8 \mathrm{~Hz}$. Randomly perturbing the phase of the grating at $8 \mathrm{~Hz}$ had little effect on contrast discrimination when the grating was also interrupted at $8 \mathrm{~Hz}$ by a uniform field (solid squares). Randomly perturbing the position of the grating at $8 \mathrm{~Hz}$ when that grating was continuously exposed made it more difficult to detect the $8-\mathrm{Hz}$ pulsed contrast increments (open circles).

than with a single phase did not lead to significantly worse performance.

This manipulation, however, produced a large effect in the continuous condition (open circles). Thresholds nearly doubled when the grating was randomly shifted eight times throughout each display interval. Notice also in Figure 5 that we replicated the continuous/interrupted effect observed previously in Experiments 1 and 2. The data in the fixed conditions of Experiment 3 show that thresholds were approximately $0.2 \log$ units higher when the background contrast was periodically interrupted at $8 \mathrm{~Hz}$ with a uniform field.

The predictions of the contrast gain control model failed in this experiment. Thresholds should have been equal in the two continuous conditions (fixed and random), but they were not. What do the three conditions shown in Figure 5 with the highest thresholds have in common, and how do they, in turn, differ from the one condition with the lowest threshold? It is not simply forcing the observer to monitor the grating as it appears in a new position every $125 \mathrm{msec}$ that causes performance to deteriorate, because only two of the three conditions with the highest thresholds have this in common. It is not simply interrupting every $125 \mathrm{msec}$ with a uniform field that causes performance to deteriorate, because once again only two of these three conditions have this in common. Instead, the one thing that all of these conditions have in common is that the periods during which the base or incremented contrasts were presented were in close temporal proximity to stimulation that was different. We will return to possible explanations for this effect in the General Discussion, below.

\section{EXPERIMENT 4}

The results above showed that interrupting the base contrast grating with a lower (zero) contrast field or with an equal contrast grating that was randomly shifted in phase at $8 \mathrm{~Hz}$ interfered with contrast discrimination. To complete this initial study of contrast gain control, it is necessary to interrupt the base contrast grating with a grating of the same spatial frequency at a higher contrast. Adjusting contrast gain to the time-averaged contrast in the field should lead also to poorer contrast discrimination in this case, because the time-averaged contrast is above the base contrast to which the increment is being 


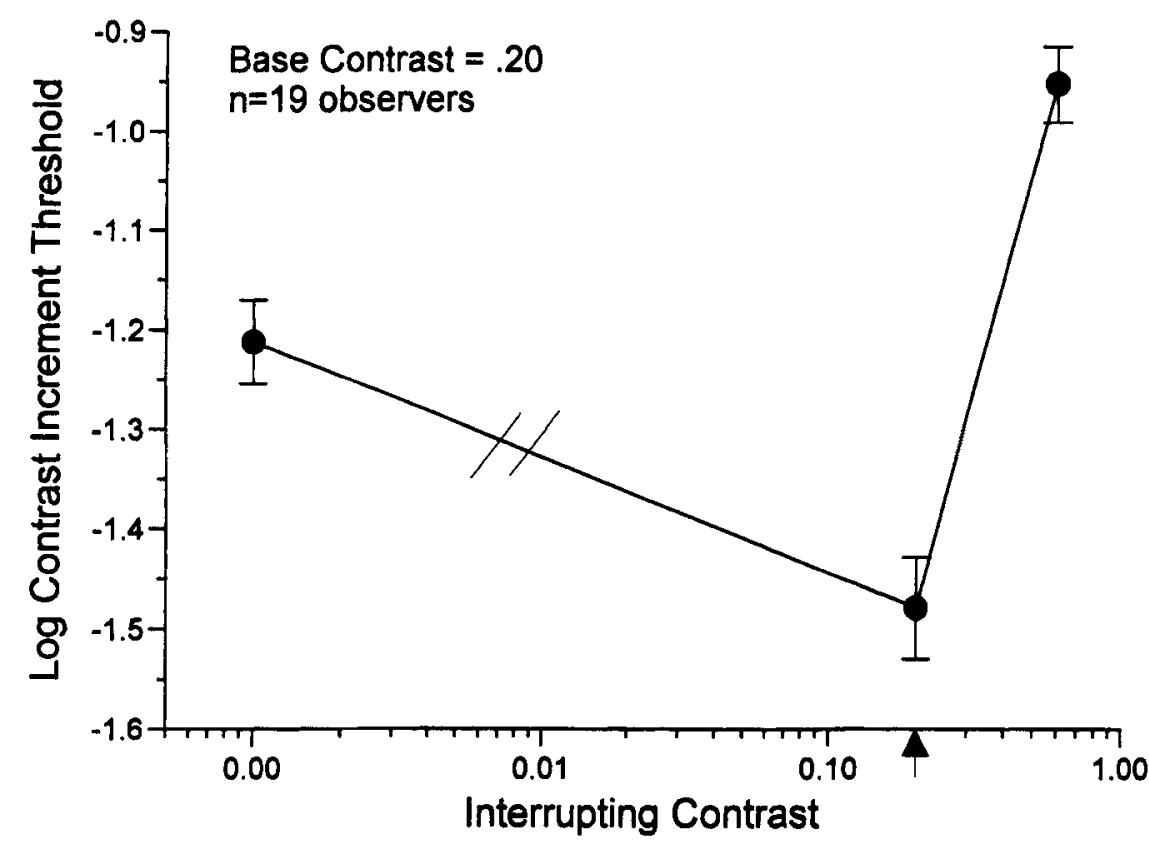

Figure 6. Average contrast increment thresholds and $S E M$ from Experiment 4. The $x$-axis shows the contrast used to interrupt the base contrast, and the $y$-axis shows the log of the contrast increment threshold. The base contrast was always 0.20 (shown by the arrow on the $x$ axis). The data point plotted above the interrupted contrast of 0.20 represents threshold in the continuous condition. Performance was significantly worse, whether the base contrast was interrupted with a lower (zero) or a higher $(0.60)$ contrast grating.

added. This manipulation also allowed us to compare our results with those from more standard contrast-masking studies using single, high-contrast forward masks.

\section{Method}

Subjects. Nineteen college-age subjects provided one threshold estimate in each of the three conditions described below. All were naive as to experimental purpose. All the observers had normal or corrected-to-normal visual acuity and wore their corrections during testing. The observers were given practice trials to acquaint them sufficiently with the procedure.

Apparatus and Stimuli. Vertical gratings were displayed as described above. The base contrast of the 0.8 -cpd gratings was fixed at 0.20 .

Design and Procedure. Threshold estimates were obtained from each observer in three conditions: two conditions in which the base contrast was interrupted and one condition in which the base contrast was presented continuously throughout each 1 -sec test interval. The grating was interrupted with contrasts of either 0.0 or 0.60 . In the latter interrupted condition, the higher contrast grating had the same spatial frequency, orientation, and spatial phase as the base contrast $(0.20)$ grating. The contrast gain control model predicts worse contrast discrimination performance in the two interrupted conditions.

\section{Results and Discussion}

The average contrast increment thresholds for each of the three conditions are plotted in Figure 6 . The $x$-axis shows the contrast presented during the off-periods of each half cycle. The base contrast was 0.20 , so the threshold plotted above an "interrupted" contrast of 0.20 represents performance in the continuous condition. It is evident that discrimination was considerably worse in both of the interrupted conditions; thresholds were higher both when the base contrast grating was interrupted with a lower (zero) contrast grating and when it was interrupted with a higher contrast grating. The contrast gain control model predicts that interrupting the base contrast grating with any contrast level that is different than the base contrast will force a shift in the CRF and produce poorer contrast discrimination. These results are consistent with that prediction.

It is also evident that discrimination was significantly worse when the base contrast grating was interrupted with a higher contrast grating than when it was interrupted with a uniform field. The time-averaged contrast in the former condition was 0.40 , and, in the latter condition, it was 0.10 . Relative to the base contrast of 0.20 , both of the time-averaged contrasts in the interrupted conditions differed from the base by a factor of 2.0. The greater effect of the high-contrast grating could be due to a difference in the time course of contrast gain for low- and high-contrast stimuli and/or asymmetry in the shape of the CRF. Our data do not allow us to discriminate between these two alternatives.

\section{GENERAL DISCUSSION}

The results of these experiments are compatible with the hypothesis that interrupting exposure to the back- 
ground contrast grating interferes with a contrast gain control mechanism, which, in turn, causes contrast discrimination to get worse. In Experiments 1 and 2, the background grating was interrupted at $8 \mathrm{~Hz}$ by a uniform field. In Experiment 3, the background grating was interrupted at $8 \mathrm{~Hz}$ by a randomly phase-shifted version of itself. In Experiment 4, the base contrast grating was interrupted with a higher contrast version of itself. In Experiments 1,2 , and 4 the time-averaged contrast in the visual field was different in the interrupted conditions than it was in the continuous conditions. If the contrast gain control signal were temporally low pass with an integration time greater than $62.5 \mathrm{msec}$, these results would be interpretable by arguing that the contrast gain was set to a value other than the value of the base contrast to which the contrast increment was applied. Only the results of Experiment 3 do not fit neatly into this model. We return to those results below.

As we noted above, models of contrast gain control include exactly this type of temporal integration stage. The time constant of this integration is left unspecified in Heeger's (1992) model. It is approximately $60 \mathrm{msec}$ in Wilson and Humanski's (1993) model, with the integration process taking approximately $200 \mathrm{msec}$ to reach $96 \%$ completion, and it required approximately $150 \mathrm{msec}$ in Wilson's (1993) masking data to be fully effective. Neurophysiological evidence (Bonds, 1993; Geisler \& Albrecht, 1992) also suggests a fast contrast gain control process that takes at least several hundred milliseconds to reach full strength. Other fast, multiplicative gain control mechanisms that are important in governing the visual system's sensitivity to contrast have also been postulated (Hayhoe, Benimoff, \& Hood, 1987; Olson, TulanayKeesey, \& Saleh, 1993). The net effect of such a process is to shift the contrast versus response function of the neuron to match the prevailing contrast in the visual field. This effectively positions a steeper part of this curve over intermediate and high contrasts after the gain control mechanism has "kicked in." Positioning a steeper part of the contrast versus response curve over intermediate and high contrasts means that a smaller contrast increment will be necessary for evoking a criterion change in responding from that neuron (see also Wilson, 1993).

The results of Experiment 3 did not fit as neatly as the other results into this contrast gain control explanation. Shifting the phase of the grating on every cycle produced an effect that was approximately equal to the effect obtained by interrupting the base contrast at $8 \mathrm{~Hz}$ with a uniform field. Why should shifting the phase of a grating at $8 \mathrm{~Hz}$ also cause a deterioration in contrast discrimination? The time-averaged contrast in the visual field remains constant in this condition, so a contrast-set gain control mechanism should adjust to this condition, just as it adjusts to the continuous conditions in which the target remained fixed in phase throughout the test interval. There are at least three explanations for this effect. One possibility is that it reflects an additional effect of pointwise luminance adaptation. The second is that it reflects the tuning of the contrast normalization or gain control networks. The third is that apparent motion may have made it difficult to detect the contrast increments in Experiment 3 . We consider these explanations in turn.

In the continuous conditions of Experiments 1, 2, and 4 and in the continuous-fixed condition in Experiment 3 , the base contrast grating is on continuously in the same position throughout each 1 -sec display interval. The target interval contains pulses of higher contrast over this base contrast. With steady fixation, local luminance adaptation might produce better sensitivity in the continuous conditions than in the interrupted conditions because observers may have been able to detect the contrast increments as pulses of intensity over the intensity produced by the base contrast at the position of the peak in the grating. Bowen and Wilson (1994) have shown that local luminance adaptation plays a role in pattern masking. Several aspects of our data argue that local luminance adaptation cannot be the only explanation for the pattern of results that we observed across these four experiments. First, if these data reflected the effects of local luminance adaptation, one would expect contrast discrimination functions in the continuous conditions of Experiment 2 at suprathreshold contrasts to show slopes near 1.0 on a double logarithmic plot, because intensity discrimination exhibits Weber's law- $\Delta I$ proportional to $I$. The slopes in the continuous condition in Experiment 2 were substantially less than 1.0 , indicating that the threshold $\Delta I$ at the peak of the grating was decreasing as a proportion of the peak intensity as the base contrast increased. Second, the contribution of pointwise luminance adaptation to masking that Bowen and Wilson observed never produced threshold differences greater than a factor of 1.5 to 2 , and these effects generally were completed within 50-75 msec. In our Experiment 4, thresholds were elevated on the average by a factor of 3.5 when the base contrast alternated with a higher contrast grating, and these effects occurred with off-period durations of $62.5 \mathrm{msec}$. We cannot rule out completely the contribution of local luminance adaptation to our results, but, similar to Bowen and Wilson's pattern-masking results, an additional contrast gain control mechanism helps to explain the results that we observed.

The second explanation for the worse performance in Experiment 3 when the grating was jittered in phase on every cycle is that it reflects the tuning of the contrast normalization network or gain control process. This tuning is supposed to be broadband; contrast in any channel can set the gain in other channels (Foley, 1994; Ross \& Speed, 1991). But numerous masking studies also show that threshold elevation is most pronounced when the target and mask are identical or at least similar (see, e.g.. Ross \& Speed, 1991; Wilson, 1993). If this masking partly reflects the effects of contrast gain control, this would imply that gain control processes show a bias; gain is set in a channel more strongly by the neurons contributing to that channel than by neurons outside that channel. If this were also true of phase (e.g., on-center vs. off-center, 
even- vs. odd-symmetric fields), the rapid periodic shifts in phase may have interfered with gain control. The target contrast increments were delivered $31.25 \mathrm{msec}$ after a new phase was introduced. The earlier cycles of contrast within each $1-\mathrm{sec}$ test period may not have been as effective in normalizing the contrast responses of the channels detecting the target in the later cycles. There have also been suggestions of mutual inhibition between opposite contrast-polarity channels (Bowen \& Wilson, 1994), so the masking that we observed in Experiment 3 may also reflect such effects.

A third potential explanation for the results of Experiment 3 relies on potential masking effects of apparent motion. The apparent motion that results from phase differences between forward maskers and targets has been shown to facilitate threshold contrast detection (Georgeson, 1988). Recall, however, that our task was contrast discrimination, not contrast detection. It is possible that the apparent motion (noted by our observers) in Experiment 3 may have made it difficult to detect the contrast increments that were delivered $31.25 \mathrm{msec}$ after the phase shifts that induced apparent motion.

We must also note that the conclusions from the first two experiments could be criticized on the grounds that the results are explained more simply by uncertainty than by invoking the temporal characteristics of contrast gain control (Pelli, 1985). Recall that the observers in the first two experiments had more exposure and access to the characteristics of the gratings in the continuous conditions than in the interrupted conditions, because, in the interrupted conditions, half of each interval contained only a uniform field. This uncertainty effect would be expected to be small or absent at low base contrasts and more pronounced at higher base contrasts, when the grating was above its own contrast threshold. This is what we found in Experiment 2. The results of Experiments 3 and 4 , however, argue that uncertainty cannot be the whole explanation for our results. In these experiments, the base contrast $(0.20)$ grating was interrupted with either a phase shift or a higher contrast $(0.60)$ grating, giving the observer as good or even better information about its spatial frequency or location. Yet contrast discrimination was worse under these conditions. The pattern of results across these experiments is predicted and explained better by postulating the operation of contrast gain control than it is by invoking uncertainty. Barlow, Kaushal, Hawken, and Parker (1987) also argued that contrast discrimination near threshold levels of base contrast cannot be explained by uncertainty.

We considered and dismissed two other potential explanations of these results. First, observers might use the contrast amplitude modulation as a cue to which interval contains the larger base contrast. The same cue is available, however, in many of the previous studies of contrast discrimination, whenever a two-temporal-alternative forced-choice procedure is used, and has been shown to be ineffective (see, e.g., Legge, 1981). Furthermore, this alternative does not fit the results of the random, contin- uous condition of Experiment 3, in which the contrast increment pulse was delivered $31.25 \mathrm{msec}$ after the grating had switched to its new phase. In other words, the contrast pulse was presented on top of the base grating, just at it was in all the other experiments, and not simultaneously with the phase shift. If the observers were using the depth of the contrast modulation in our task, they should have been just as able to do this in the random, continuous condition as in the fixed, continuous condition, but the data show that they were not.

Second, the temporal bandpass character of transient mechanisms may explain our findings. In the interrupted conditions, the increment (at $8 \mathrm{~Hz}$ ) might be masked by the spatiotemporal energy associated with the interrupted display (also $8 \mathrm{~Hz}$ ). The literature, however, clearly indicates that the temporal frequency of a mask does not affect threshold elevation of a brief target. For example, Foley and Boynton (1993) found no difference in the threshold elevation for detecting a 33-msec, 2-cpd grating with masks ranging from 0 to $15 \mathrm{~Hz}$. Additionally, in an adaptation study, Wilson and Humanski (1993) cast doubt on a sustained/transient account of such results. They showed that the threshold elevation for a $16.7-\mathrm{Hz}$ test stimulus was nearly equal to the threshold elevation for a 500-msec test, but both were substantially higher than the threshold elevation for a $30-\mathrm{msec}$ test after adapting to a $1.5-\mathrm{Hz}$ masker. These results do not fit neatly into detection based on the sustained/transient dichotomy. Indeed, Wilson and Humanski interpreted their results in terms of the effects of contrast gain control.

Why should the effects of gain control on contrast discrimination be less pronounced at higher spatial frequencies, as we observed in the first experiment? One explanation for this effect relies on the same argument as that used to explain the masking effects that we observed in Experiment 3 when phase was randomly shifted on every cycle. Small eye movements would introduce larger phase shifts across the course of a 1-sec display period with 3.2 -cpd gratings than with 1.6- and 0.8 -cpd gratings. Such random phase shifts across cycles would reduce the effectiveness of gain control adjustments to the base contrast, similar to the effects that we observed in Experiment 3 with deliberate phase shifts. Gain control may be less effective in maximizing contrast discrimination at higher spatial frequencies because the inadvertent phase shifts introduced by small eye movements interfere with contrast normalization in phase-tuned channels.

Finally, we note that contrast gain control might operate as response-set or contrast-set gain control (Foley \& Boynton, 1993). Response-set gain control would be realized by adjusting the maximum response of neurons downward on the basis of prior strong responding-a sort of adaptation-as-fatigue model. Our results in these experiments, using a zero-contrast interrupting mask, argue against response-set gain control. One would expect little response to the zero-contrast interrupting mask. With a contrast-set gain control model, however, and with some temporal averaging of contrast in the gain control 
network, one would expect a shift in the CRF to lower contrast levels, with concomitantly poorer contrast discrimination at the base contrast level.

In conclusion, interrupting a suprathreshold grating at $8 \mathrm{~Hz}$, with a uniform field, with a phase-shifted version of that same grating, or with a higher contrast grating at the same spatial frequency, resulted in poorer contrast discrimination relative to conditions in which the grating was exposed continuously for $1 \mathrm{sec}$ and the contrast increment was pulsed at $8 \mathrm{~Hz}$. These effects held only at lower spatial frequencies $(<1.6 \mathrm{cpd})$. The results may be explained by assuming that contrast discrimination reflects the visual system's degree of adjustment to the base contrast; that is, contrast gain ( $\Delta$ Response/ $\Delta$ Contrast) is adjusted to be at a maximum around the prevailing contrast. The networks that perform this contrast normalization are assumed to average the contrast in the visual field over time. When this time-averaged contrast is different from the base contrast to which the contrast increment is applied, contrast discrimination suffers.

\section{REFERENCES}

Albrecht, D. G., \& Geisler, W. S. (1991). Motion selectivity and the contrast-response function of simple cells in the visual cortex. Visual Neuroscience, 7, 531-546.

Barlow, H. B., Kaushal, T. P., Hawken, M., \& Parker, A. J. (1987). Human contrast discrimination and the threshold of cortical neurons. Journal of the Optical Society of America A, 4, 2366-2371.

BONDS, A. B. (1989). Role of inhibition in the specification of orientation selectivity of cells in the cat striate cortex. Visual Neuroscience, 2, 41-55.

Bonds, A. B. (1993). The encoding of cortical contrast gain control. In R. Shapley \& D. M.-K. Lam (Eds.), Contrast sensitivity (pp. 215-230). Cambridge, MA: MIT Press.

BOWEN, R. W., \& WILSON, H. R. (1994). A two-process analysis of pattern masking. Vision Research, 34, 645-657.

Fol.EY, J. M. (1994). Human luminance pattern-vision mechanisms: Masking experiments require a new model. Journal of the Optical Society of America A, 11, 1710-1719.

FOLEY, J. M., \& BOYNTON, G. M. ( 1993). Forward pattern masking and adaptation: Effects of duration, interstimulus interval, contrast, and spatial and temporal frequency. Vision Research, 33, 959-980.

Geisler, W. S., \& AlBRECHT, D. G. (1992). Cortical neurons: Isolation of contrast gain control. Vision Research, 32, 1409-1410.

Georgeson, M. A. (1988). Spatial phase dependence and the role of motion detection in monocular and dichoptic forward masking. $\mathrm{Vi}$ sion Research, 28, 1193-1205.

Greenlee, M. W., \& Heitger, F. (1988). The functional role of contrast adaptation. Vision Research, 28, 791-797.

Hayhoe, M. M., Benimoff, N. I., \& Hood, D. C. (1987). The timecourse of multiplicative and subtractive adaptation processes. Vision Research, 27, 1981-1996.

HEEger, D. J. (1992). Normalization of cell responses in cat striate cortex. Visual Neuroscience, 9, 181-197.

HEEGER, D. J. (1994). The representation of visual stimuli in primary visual cortex. Current Directions in Psychological Science, 3, 159-163.

LEGGE, G. E. (1981). A power law for contrast discrimination. Vision Research, 21, 457-467.

Olson, J. D., Tulanay-Keesey, U., \& Saleh, B. E. A. (1993). Fading time of retinally-stabilized images as a function of background luminance and target width. Vision Research, 33, 2127-2137.

PELLI, D. G. (1985). Uncertainty explains many aspects of visual contrast detection and discrimination. Journal of the Optical Society of America A, 2, 1508-1531.

Ross, J., \& SPEED, H. D. (1991). Contrast adaptation and contrast masking in human vision. Proceedings of the Royal Society of London: Series $B, 246,61-69$.

Tolhurst, D. I., \& HeEger, D. J. (1997). Contrast normalization and a linear model for the directional selectivity of simple cells in cat striate cortex. Visual Neuroscience, 14, 19-25.

Wilson, H. R. (1993). Nonlinear processes in visual pattern discrimination. Proceedings of the National Academy of Sciences, 90, 9785-9790.

WiLSON, H. R., \& HUMANSKI, R. (1993). Spatial frequency adaptation and contrast gain control. Vision Research, 33, 1133-1149.

\section{NOTE}

1. We computed the slope using only the three highest contrasts because the curve is approximately linear on a $\log -\log$ plot only at contrast levels greater than two to three times threshold contrast (Wilson \& Humanski, 1993). Threshold contrasts were .02 to .03 , so we computed the slopes starting at a background contrast of .06 .

(Manuscript received April 4, 1997; revision accepted for publication September 17, 1997.) 